\title{
The influence of diffusion and mass loss on the chemical composition of subdwarf B stars
}

\begin{abstract}
K. Unglaub and I. Bues
Dr. Remeis-Sternwarte Bamberg, Astronomisches Institut der Universität Erlangen-Nürnberg, Sternwartstraße 7, 96049 Bamberg, Germany

Received 15 March 2001 / Accepted 3 May 2001

Abstract. We investigate the influence of diffusion and mass loss on the chemical composition in subdwarf B stars in the range $25000 \mathrm{~K} \leq T_{\text {eff }} \leq 35000 \mathrm{~K}, 5.5 \leq \log g \leq 6.0$. Within the outer hydrogen-rich envelope characterized by mass $<10^{-2} M_{*}$ for the elements $\mathrm{H}, \mathrm{He}, \mathrm{C}, \mathrm{N}$ and $\mathrm{O}$ the equations of continuity, the momentum equations and the equation of radiative transfer are solved simultaneously. For various mass loss rates the time evolution of the chemical composition is predicted within time scales of $\approx 10^{8} \mathrm{yr}$, which correspond to the typical lifetimes of the sdB's near the Extended Horizontal Branch. According to the results weak winds with $\dot{M} \approx 10^{-13} M_{\odot} / \mathrm{yr}$ may explain the typical helium deficiencies by more than one order of magnitude in the atmospheres of these stars. Winds with $10^{-14} M_{\odot} / \mathrm{yr}<\dot{M}<10^{-13} M_{\odot} / \mathrm{yr}$ may lead to strong deficiencies as well as to enrichments of the CNO elements. The composition in the outer envelope changes in time scales similar to the typical lifetimes of the sdB's. From estimates of the radiative acceleration in the wind region, which make the existence of weak winds plausible, and from a comparison with the case of hot white dwarfs we suggest that the abundance anomalies observed in sdB stars are related to the combined effects of diffusion and mass loss.
\end{abstract}

Key words. diffusion - stars: abundances - stars: subdwarfs - stars: white dwarfs - stars: evolution

\section{Introduction}

The hot subluminous B stars (in this paper no distinction will be made between the $\mathrm{sdB}$ and sdOB spectroscopic classes) can be identified with models of the extreme Horizontal Branch (EHB; Heber 1986; Saffer et al. 1994; for a review about these stars see Heber 1998). Their stellar parameters are typically in the range $25000 \mathrm{~K} \leq$ $T_{\text {eff }} \leq 40000 \mathrm{~K}, 5 \leq \log g \leq 6$, their total masses are $\approx 0.5 M_{\odot}$. According to Dorman et al. (1993) the internal structures of EHB models are characterized by an almost exhausted hydrogen burning shell with a mass below about $0.02 M_{\odot}$. During a time scale of about $10^{8}$ yr $T_{\text {eff }}$ and $\log g$ remain nearly unchanged. Because of their thin hydrogen shell, after helium core exhaustion EHB stars fail to ascend the asymptotic Giant Branch (AGB) and evolve directly into white dwarfs.

In the atmospheres of sdB stars helium is typically deficient by more than one order of magnitude, but there is a wide spread between approximately solar abundances or even overabundances in a few cases and stars with $\mathrm{He} / \mathrm{H}<10^{-4}$ (e.g. Moehler et al. 1990; Saffer et al. 1994; Maxted et al. 2001; Edelmann et al. 2001). The helium deficiency usually is accompanied by abundance anoma-

Send offprint requests to: K. Unglaub,

e-mail: unglaub@sternwarte. uni-erlangen.de lies of heavy elements, which has been shown by several model atmosphere analysis of sdB's (Baschek et al. 1982a,b; Heber et al. 1984, 1999, 2000; Lamontagne et al. $1985,1987)$. In some cases various heavy elements are deficient by more than a factor of 100 , whereas others have abundances close to the solar value. It is remarkable that from the CNO elements nitrogen is pronouncedly less reduced than carbon and oxygen in most cases. In the past, several attempts have been made to explain these results with the assumption of an equilibrium between gravitational settling and radiative levitation (e.g. Michaud et al. 1989; Bergeron et al. 1988; Ohl et al. 2000). Although in some cases these calculations lead to coincidences between predicted and observed values, it has not been possible to reproduce all abundances simultaneously. Especially the predicted abundances of helium are in general too low. Therefore Fontaine \& Chayer (1997) investigated the effect of an additional weak stellar wind on helium in sdB's. The results show, that this model may explain the observed abundances, if the mass loss rates are in the range between about $10^{-14}$ and $10^{-13} M_{\odot} / \mathrm{yr}$. This gives rise to the question, how the heavy elements are affected in this diffusion and mass loss scenario. Is it possible to explain not only the abundances of helium, but in addition the anomalies of heavy elements? It is the main intention of 
the present paper to investigate this for the elements $\mathrm{C}$, $\mathrm{N}$ and $\mathrm{O}$.

Recently, several sdB stars have been found to be pulsating (termed EC 14026 stars after the prototype, see O'Donoghue et al. 1999 for a review). The existence of pulsating sdB stars was predicted by Charpinet et al. (1996). The driving region is associated with an opacity bump in mass depths of $10^{-9}$ to $10^{-10} M_{*}$, which is preferably due to the opacity of iron. However, the existence of unstable pulsation modes requires a metal enrichment in the driving region. Subsequently, Charpinet et al. (1997) carried out equilibrium diffusion calculations for iron and indeed predicted overabundances over a substantial fraction of the outer envelope. Although we do not take into account iron, in the present paper we will discuss the possibility of metal enrichments in the presence of mass loss.

In Unglaub \& Bues (2000; henceforth Paper I) the effect of diffusion and mass loss in hot white dwarfs has been investigated. In the present paper, the method used in Paper I is applied to the case of sdB's. In Sect. 2 the most important assumptions are summarized. In Sect. 3 the results for $T_{\text {eff }}=35000 \mathrm{~K}, \log g=5.5$ and several mass loss rates are discussed in detail. Then some results for other stellar parameters are presented. In Sect. 4 the existence of weak winds is discussed and the situation in sdB's is compared with the one in hot white dwarfs, for which according to Paper I the predictions of the diffusion/mass loss model are in satisfactory agreement with the observational data. In Sect. 5 the results are summarized and the conclusions are discussed.

\section{Assumptions and computational method}

In the present investigation, we intend to predict the time evolution of the surface composition for given values of $T_{\text {eff }}, \log g$ and mass loss rates $\dot{M}$. A total stellar mass $M_{*}=0.5 M_{\odot}$ is assumed in all cases. The calculations start with solar composition in all depths. As in Paper I, a fixed gas pressure scale is used in the range $2000 \mathrm{~Pa} \leq P_{\mathrm{g}} \leq 10^{16} \mathrm{~Pa}$. This computation domain covers the regions between the stellar atmosphere and mass depths of the order $10^{-2} M_{*}$ (for $\log g=5.5$ ). Nuclear burning and the presence of an underlying helium-rich region are not taken into account. This implies the assumption that the surface composition can be predicted independently of the mass of the outer hydrogen-rich envelope. It is justified, if the total mass loss is small in comparison to the hydrogen layer mass and if the diffusion time scales at the lower boundary are large in comparison to the time scales of stellar evolution. In Sect. 3.2 this point will be discussed in more detail.

The temperature structure is obtained from

$\frac{\mathrm{d} T}{\mathrm{~d} r}=-\frac{3}{16} T_{\mathrm{eff}}^{4} \bar{\chi} \frac{1}{T^{3}} \frac{R_{*}^{2}}{r^{2}}$

with $T^{4}=\frac{1}{2} T_{\text {eff }}^{4}$ at the outer boundary. $R_{*}$ is the stellar radius and $r$ is the distance from the stellar center.
To account for the effect of the composition on the temperature strucure, we calculate monochromatic continuum opacities for 264 wavelengths in the range $0.1 \AA \leq \lambda \leq$ $10000 \AA$. Electron scattering and the contributions of all ions with their absorption edges are taken into account with the exception of neutral and singly ionized C, N and O. For hydrogen-like ions the bound-free Gaunt factors from Karzas \& Latter (1961) are used, for the other ions the absorption cross sections according to Clark et al. (1986). From the monochromatic continuum opacities the Rosseland mean opacity $\bar{\chi}$ is evaluated. Equation (1) is a sufficient approximation for our purposes. For the mass loss rates of interest the matter of the stellar atmosphere is removed within a few years or even less. Diffusion cannot change the composition significantly, before the atmosphere is replaced by matter from underlying regions. Therefore in diffusion calculations with mass loss especially the conditions in deeper regions are important. This is in contrast to calculations without mass loss, where the surface abundance can be predicted only if the radiative accelerations in the stellar atmosphere are known.

The diffusion velocities $v_{\mathrm{d}}$ are calculated as in Unglaub $\&$ Bues (1997) from the system of linear equations derived by Burgers (1969), which consists of the various momentum equations and the condition of zero net mass flow. For an element $l$ the momentum equation reads

$$
\begin{array}{r}
-\frac{\mathrm{d} p_{1}}{\mathrm{~d} r}-n_{\mathrm{l}} m_{\mathrm{l}} g_{(r)}+n_{\mathrm{l}} \bar{Z}_{\mathrm{l}} e E+n_{\mathrm{l}} F_{\mathrm{l}, \mathrm{rad}} \\
=\sum_{t} K_{\mathrm{l}, \mathrm{t}}\left(v_{\mathrm{d}, \mathrm{t}}-v_{\mathrm{d}, \mathrm{l}}\right) .
\end{array}
$$

The expressions on the left represent the momentum per unit volume and unit time transferred to the element by the gradient of the partial pressure, gravity, the electric field $E$ and the radiative force $F_{1, \mathrm{rad}}$.

The radiative forces for the various elements are calculated as in Unglaub \& Bues (1996) with the improvements as in Unglaub \& Bues (1998).

The momentum transfer from the photons to the various elements via bound-bound and bound-free transitions is taken into account. To obtain the bound-bound contribution, the sum of the continuum opacity and the line opacity of the line under consideration is used to derive the monochromatic flux from the diffusion approximation. The effect of flux blocking, this is the possible overlap of several lines, is neglected. As will be explained below, a few lines only contribute significantly to the radiative acceleration, therefore this effect seems to be of minor importance. The line list consists of 280 lines and is similar to Vauclair et al. (1979). The radiative acceleration due to lines is of importance especially in those depths, in which the preferred ionization stage of $\mathrm{C}, \mathrm{N}$ and $\mathrm{O}$ changes from helium- to hydrogen-like. For carbon this requires temperatures of the order $5 \times 10^{5} \mathrm{~K}$. Outwards there is a wide range of depths, where all these elements have heliumlike configuration. Here the radiative acceleration is very low, because the flux maximum is at wavelength ranges far away from the resonance lines of the helium-like ions. 
Lower ionizations states are of importance in the outer regions only, where the temperature is lower than about $10^{5} \mathrm{~K}$. There, however, for the mass loss rates considered in the present paper, the influence of diffusion on the results is small, because the mass within these regions is lost within less than about $10^{4} \mathrm{yr}$. Thus only the radiative acceleration for helium- and hydrogen-like ions is of major importance for the results. In addition, the main contribution is due to a few strong resonance lines. Therefore we do not believe that a more complete line list could significantly change the results. To obtain the radiative acceleration due to bound-free transitions, the factor $f_{\text {ion }}$ from Massacrier (1996) is taken into account. This factor prescribes how in photoionization processes the momentum is shared between the photoelectron and the heavy particle.

The dependence of the gravity $g$ on the distance $r$ from the stellar center is approximated by $g_{(r)} \approx \frac{G M_{*}}{r^{2}}$. The resistance coefficients $K_{1, \mathrm{t}}$ are obtained according to Paquette et al. (1986a). The charge $\bar{Z}_{1}$ of an element is the mean value over all ionization states. The ionization equilibrium is calculated as in Unglaub \& Bues (1996), where the partition functions of the various ions have been evaluated by use of the cutoff procedure. Thermal diffusion and ionization effects have been neglected. Paquette et al. $(1986 \mathrm{a}, \mathrm{b})$ have shown that for plasma conditions typical for hot high gravity stars thermal diffusion is at least less effective than gravitational settling. The ionization effects may be of special importance in regions with partial ionization of the elements. In the deeper regions of sdB models hydrogen and helium are preferably completely ionized, thus it should be expected that at least for these elements the effect is small. Some trace amounts of neutral hydrogen especially in excited states may still be present, however. As we neglect the interaction of charged particles with neutral hydrogen via polarization, the diffusion velocities probably tend to be somewhat too large.

The winds are assumed to be chemically homogeneous. For a radiatively driven wind, this requires that in the wind region outside of the computation domain the momentum absorbed from the photons is effectively redistributed between the various species of particles, so that all of them have the same velocity and no separation of elements is possible. This point will be addressed once more at the end of Sect. 4. Within the computation domain the wind velocity $v_{\mathrm{w}}$ is obtained from the equation of continuity:

$v_{\mathrm{w}}=\frac{\dot{M}}{4 \pi r^{2}} \frac{1}{\rho}$

where $\rho$ is the density and $\dot{M}$ the mass loss rate. $v_{\mathrm{w}}$ is the velocity of the mass flow, whereas the diffusion velocities are the velocities relative to a frame in which the mass flow is zero. Thus the total velocity of an element is the sum of $v_{\mathrm{w}}$ and the diffusion velocity.

The numerical method essentially is the monotonic transport scheme with some modifications. It has been described in detail in Paper I and used for the diffusion and mass loss calculations for hot white dwarfs. The stability of the method requires, that for the geometrical distance $\Delta r$ between two grid points the Courant condition is fulfilled: $\Delta r \leq v \Delta t$, where $v$ is the total velocity of an element and $\Delta t$ the time step. If the diffusion velocities are small in comparison to the wind velocity, which is true especially in the outer regions, this condition means that the mass $\Delta m$ between two grid points does not exceed the mass which is lost during one time step. To account for the Courant condition, in dependence of the various velocities a variable grid spacing is used. For the calculations presented in this paper, the maximum time step is $\Delta t=100 \mathrm{yr}$, except for the result shown in Figs. 5, 6 and 7 , for which $\Delta t=1000 \mathrm{yr}$ is the maximum time step. For various mass loss rates a comparison has shown that the results do not noticeably change, if $\Delta t$ is varied in this range.

\section{Results}

In Sect. 3.1 the results of these calculations are presented, which start with solar composition throughout the computation domain. The possible influence of the inner boundary condition is investigated in Sect. 3.2. It is shown under which circumstances the existence of a helium-rich layer below the hydrogen-rich envelope can change the predicted surface composition. In Sect. 3.3 we comment on the possible influence of diffusion and mass loss on the helium abundance during the post-EHB evolution.

\subsection{Results for solar initial composition}

In Fig. 1, for $T_{\text {eff }}=35000 \mathrm{~K}, \log g=5.5$ and several mass loss rates the predicted surface number fractions of the various elements are plotted as a function of time. These are the number fractions near $\bar{\tau}=1$, which are nearly constant within the stellar atmosphere for the mass loss rates considered here. Figure 1a shows the results for $\dot{M}=1.0 \times 10^{-14} M_{\odot} /$ yr. He, $\mathrm{N}$ and $\mathrm{O}$ sink continuously. Within a few millions of years the number fraction of helium drops below a value of $10^{-5}$. After about $3 \times 10^{7} \mathrm{yr}$ the abundances of $\mathrm{He}, \mathrm{N}$ and $\mathrm{O}$ are below $10^{-10}$. The number fraction of carbon decreases by somewhat more than a factor of 100 within the first two millions of years after the onset of diffusion and mass loss. Afterwards it slightly increases and is approximately constant for times in the range $10^{7}$ yr $\leq t \leq 10^{8}$ yr. So $\dot{M}=10^{-14} M_{\odot} /$ yr leads to a surface composition with carbon reduced by about a factor of 100, whereas He, $\mathrm{N}$ and $\mathrm{O}$ should not be detectable at all. The elements sink in times which are short in comparison to the typical times the sdB's spend near the EHB.

In Fig. 1b the results for $\dot{M}=2.5 \times 10^{-14} M_{\odot} /$ yr are presented. Because of the higher mass loss rate helium sinks more slowly. Now it takes about $3 \times 10^{7} \mathrm{yr}$ until the helium abundance decreases below $10^{-5}$. The time evolution of the surface composition of the CNO 

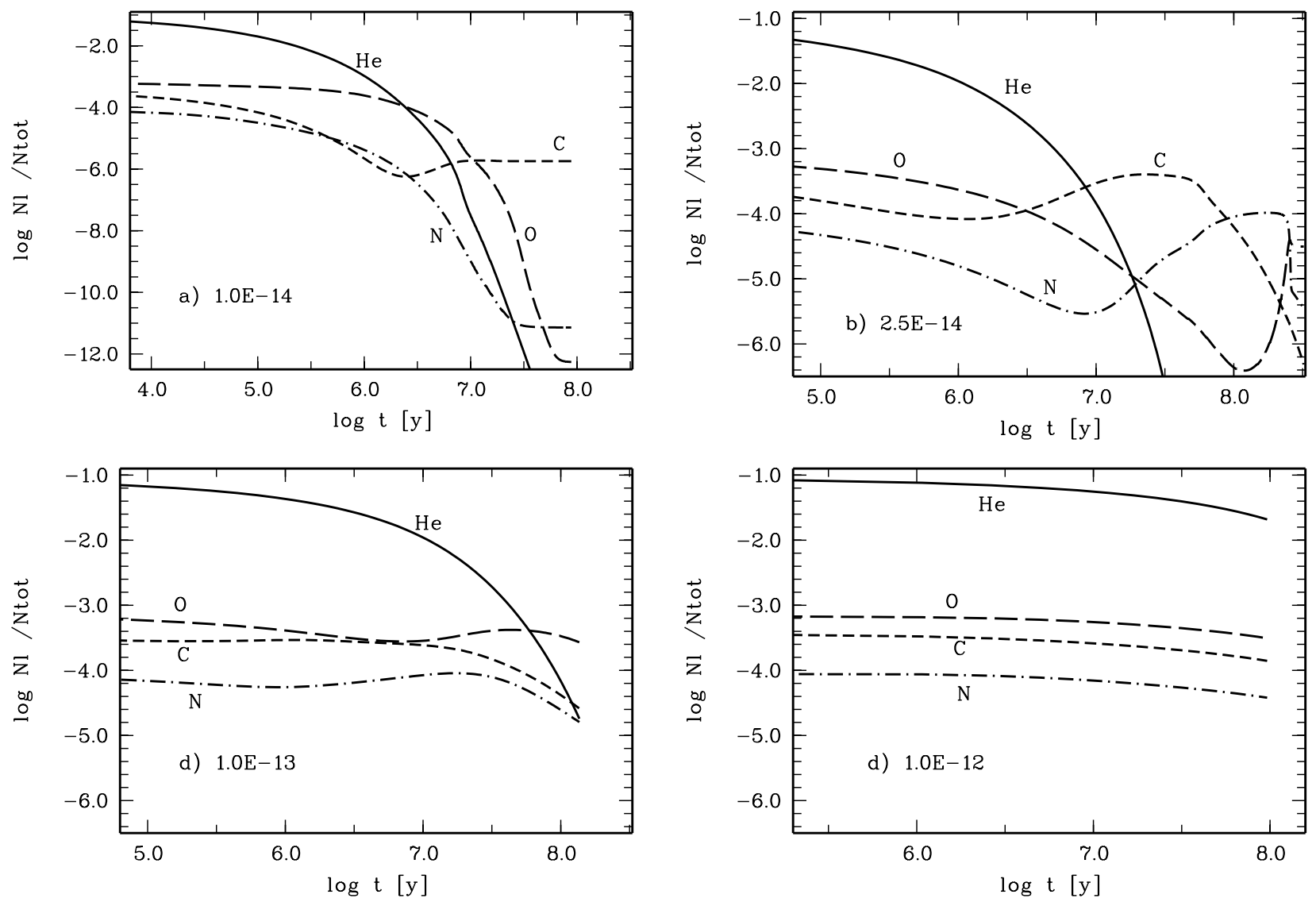

Fig. 1. Predicted surface number fractions as a function of the time for $T_{\text {eff }}=35000 \mathrm{~K}, \log g=5.5$ for various mass loss rates: a) $1.0 \times 10^{-14}$, b) $2.5 \times 10^{-14}$, c) $1.0 \times 10^{-13}$ and d) $1.0 \times 10^{-12} M_{\odot} / \mathrm{yr}$.

elements is in principle similar for each element. It can be subdivided into three stages. First, the number fraction decreases. Second, it increases to a value which may be similar or even slightly larger than the inital one. Third, the abundance decreases again. Within $10^{8} \mathrm{yr}$, for oxygen only the first stage is realized. Its abundance continuously decreases by about a factor of $10^{3}$. If, however, the calculations are continued over larger time scales, it can be seen in Fig. 1b that then the oxygen number fraction strongly increases. For nitrogen, the first stage takes about $10^{7} \mathrm{yr}$. The element is reduced by somewhat more than a factor of ten. Then its number fraction increases, so that after $\approx 10^{8} \mathrm{yr}$ approximately the solar value is recovered. After $\approx 2.5 \times 10^{8}$ yr it would begin to sink back again. The carbon abundance slightly decreases during the first million of years. Then carbon is enriched until for times larger than about $5 \times 10^{7} \mathrm{yr}$ its abundance decreases again. This behaviour of the $\mathrm{CNO}$ elements in the course of time leads to various abundance anomalies. For times $t<10^{8} \mathrm{yr}$ preferably carbon should be the most abundant element, whereas $\mathrm{N}$ and $\mathrm{O}$ are more or less depleted. After $t \approx 10^{8} \mathrm{yr}$ this picture chances. Then we expect about solar abundance of $\mathrm{N}$ accompanied by a reduction of $\mathrm{C}$ and $\mathrm{O}$. To understand these results in more detail, it is necessary to consider the various abundances as a function of depth. This will be done in Fig. 2 below.
In Fig. 1c the surface composition as a function of time is plotted for $\dot{M}=10^{-13} M_{\odot} /$ yr. For this mass loss rate helium sinks within a time scale similar to the sdB's time scales of stellar evolution. After $10^{7} \mathrm{yr}$ helium is reduced by about a factor of ten, after $10^{8} \mathrm{yr}$ its abundance has decreased to a value between $10^{-4}$ and $10^{-5}$. An effect of diffusion on the CNO elements is still present. However, it is much less pronounced than in the previous cases with lower mass loss rates. The number fractions deviate by less than a factor of ten from the initial ones. Finally, in Fig. $1 \mathrm{~d}$ we consider the case with $\dot{M}=10^{-12} M_{\odot} / \mathrm{yr}$. Here the effect of diffusion is small. Within $10^{8} \mathrm{yr}$ the number fraction of helium decreases by a factor of five, those of the CNO elements by about a factor of two only. The relative abundance of these elements remain nearly unchanged. From these results we clearly recognize the decreasing effect of diffusion on the surface abundances with increasing mass loss rate.

In Fig. 2 we consider again the case with $\dot{M}=2.5 \times$ $10^{-14} M_{\odot} / y r$. For various times the number fractions of the elements are plotted as a function of the gas pressure. Although the inner boundary of the computation domain is at $P_{\mathrm{g}}=10^{16} \mathrm{~Pa}$, the results are shown only for $P_{\mathrm{g}} \leq 10^{14} \mathrm{~Pa}$, which corresponds to a mass depth of about $10^{-3} M_{*}$. In deeper regions the composition remains nearly unchanged, because due to the low velocities 

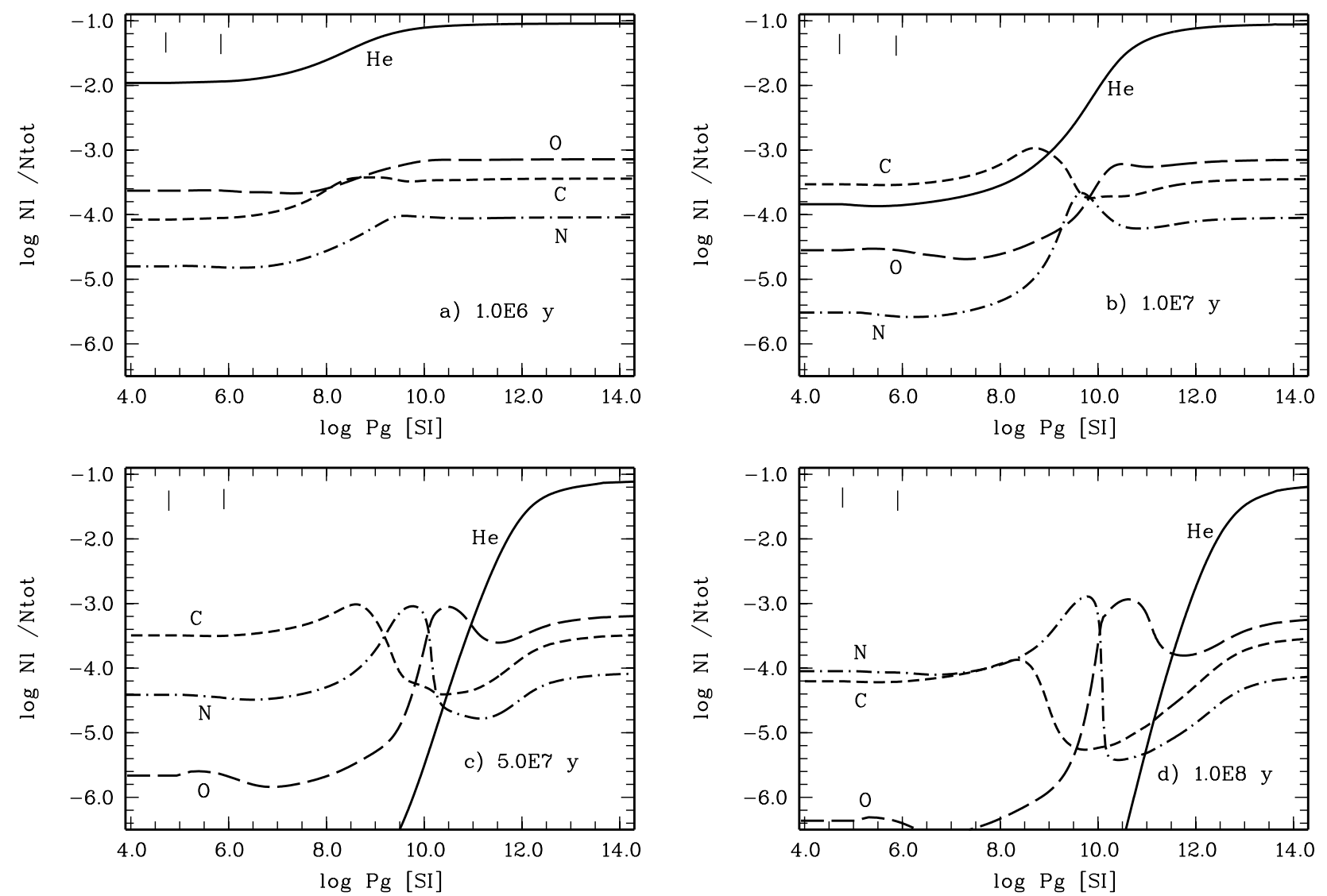

Fig. 2. Number fractions of the various elements as a function of the gas pressure for $T_{\text {eff }}=35000 \mathrm{~K}, \log g=5.5$ and $\dot{M}=2.5 \times 10^{-14} M_{\odot} /$ yr after various times: a) $10^{6}$, b) $10^{7}$, c) $5 \times 10^{7}$ and d) $10^{8}$ yr. The two tick marks in the upper part of each figure indicate the Rosseland mean optical depths $\bar{\tau}=10$ and 100 , respectively. $\bar{\tau}=1$ is at the left boundary of each figure.

Table 1. For several gas pressures $P_{\mathrm{g}}$ (in $\mathrm{Pa}$ ) the mass depths $\Delta M$ (in $M_{*}$ with $M_{*}=0.5 M_{\odot}$ ) and the temperatures $T$ (in $\mathrm{K})$ are given for a model with $T_{\text {eff }}=35000 \mathrm{~K}, \log g=5.5$ and solar composition.

\begin{tabular}{lcccccc}
\hline $\log P_{\mathrm{g}}$ & 4.0 & 6.0 & 8.0 & 10.0 & 12.0 & 14.0 \\
$\log \Delta M$ & -12.1 & -10.1 & -8.1 & -6.2 & -4.5 & -3.0 \\
$\log T$ & 4.60 & 5.07 & 5.51 & 5.92 & 6.42 & 6.84 \\
\hline
\end{tabular}

the effect of diffusion is negligible within the time scales of interest. The left boundary of each figure corresponds to a Rosseland mean optical depth $\bar{\tau}=1$. In addition, the two tick marks in the upper left part indicate values of $\bar{\tau}=10$ and 100, respectively. In addition, in Table 1 for several gas pressures the mass depth and the temperature are given for a model with solar composition in all depths (for other compositions the relation between the gas pressure and the mass depth would be very similar, whereas the temperatures may be up to about $20 \%$ lower if e.g. the abundances of helium and the heavy elements are reduced by a factor of 100). It can be seen from the various figures, that in the outer regions the composition always is in good approximation homogeneous. As the drift velocities due to mass loss are large in comparison to the diffusion velocities, no steep concentration gradients are present.
Now let us consider the various figures in detail. In Fig. 2 a the situation after $10^{6} \mathrm{yr}$ is shown. For $\log P_{\mathrm{g}} \geq 10$ the composition is still unchanged. In the outer regions the number ratios of the various elements are reduced by not more than a factor of ten and the relative abundances of the CNO elements are similar to the initial ones. This situation has changed after $10^{7}$ yr (Fig. 2b). Whereas the number fractions of $\mathrm{N}$ and $\mathrm{O}$ have further decreased, the surface abundance of carbon is now larger than after $10^{6}$ yr. To understand this result, we must recognize that at $\log P_{\mathrm{g}} \approx 8.5$ (which corresponds to a mass depth of the order $10^{-8} M_{*}$ ) a carbon reservoir has built up. Here the number fraction of carbon is about $10^{-3}$, which is larger than the initial one. This enrichment is a consequence of the radiative acceleration, which is especially effective in regions where the element has hydrogen-like configuration. Thus the total velocitiy (diffusion+wind) is large. Then, however, during their movement in outward direction the particles reach a zone where carbon has helium-like configuration. Here the radiative acceleration is small. Thus the total velocity decreases. According to the equation of continuity, this deceleration leads to an enrichment of the element. Because of the non-negligible coupling between internal and surface composition in the presence of mass loss, this finally leads to an increasing 

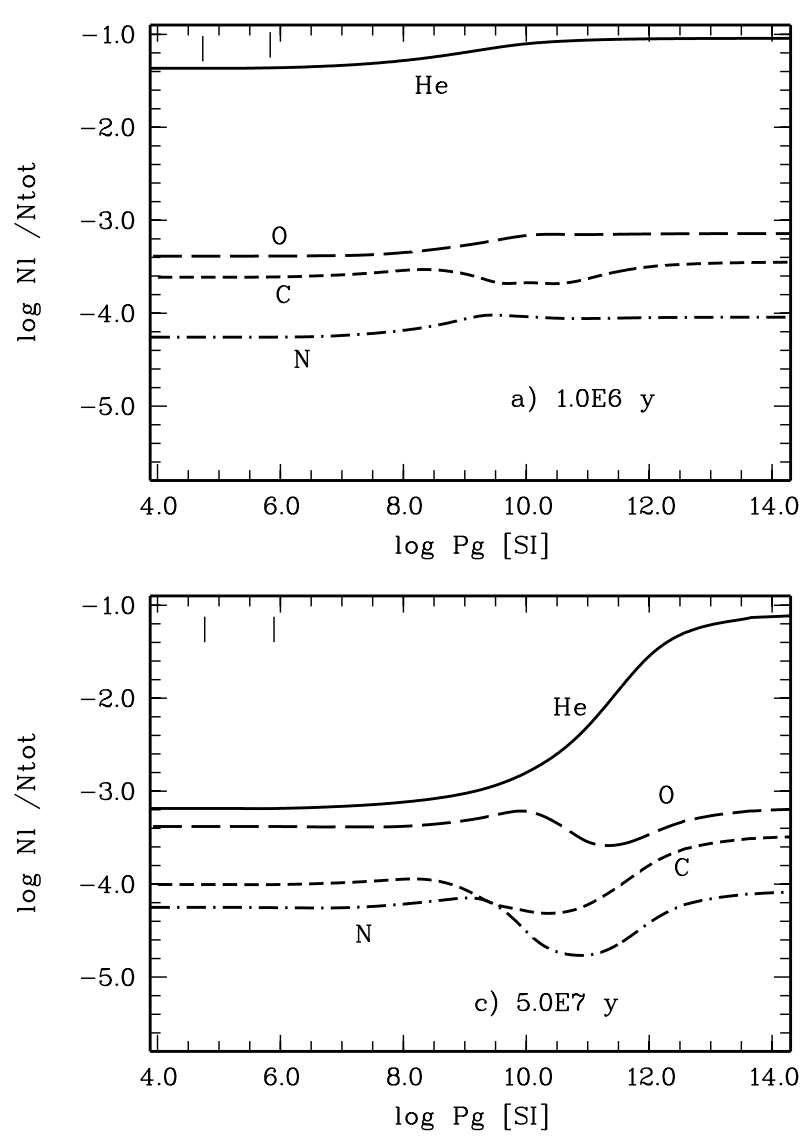

Fig. 3. The same as Fig. 2 for $\dot{M}=1.0 \times 10^{-13} M_{\odot} /$ yr.

surface abundance of carbon. For $\mathrm{N}$ and $\mathrm{O}$ the results are in principle similar, only the time scales differ. At $\log P_{\mathrm{g}} \approx 9.5$ in Fig. $2 \mathrm{~b}$ we see that a nitrogen reservoir begins to build up, whereas for oxygen the same effect cannot be clearly recognized yet. If compared to carbon, $\mathrm{N}$ and $\mathrm{O}$ have hydrogen-like configuration in deeper regions, where the diffusion velocities are lower. Therefore the formation of a reservoir of these elements takes longer. This can be seen from Fig. 2c, which shows the situation after $5 \times 10^{7}$ yr. Now, at $\log P_{\mathrm{g}} \approx 9.5$ the number fraction of $\mathrm{N}$ is about $10^{-3}$, which is a factor of ten more than the initial one. This has the consequence that the surface abundance increases, which after $5 \times 10^{7}$ yr is larger by about a factor of ten than after $1 \times 10^{7}$ yr. For oxygen, however, the surface abundance is still decreasing. This is because the corresponding reservoir is not yet sufficiently filled up to reverse this trend. Figure 2d shows the results after $10^{8} \mathrm{yr}$. The surface abundance of $\mathrm{C}$ has decreased in comparison to the previous case. This is because the reservoir near $\log P_{\mathrm{g}}=8.5$, which has still been clearly present after $5 \times 10^{7} \mathrm{yr}$, is depleted now. To understand this, we must have in mind that in the inner regions the elements are almost completely ionized and thus the radiative acceleration is negligibly small. Therefore gravitational settling is the dominating effect. As towards deeper regions the wind velocity decreases faster than the diffusion velocities, below a certain depth the total velocity will be negative. This means that no particles of the corresponding element
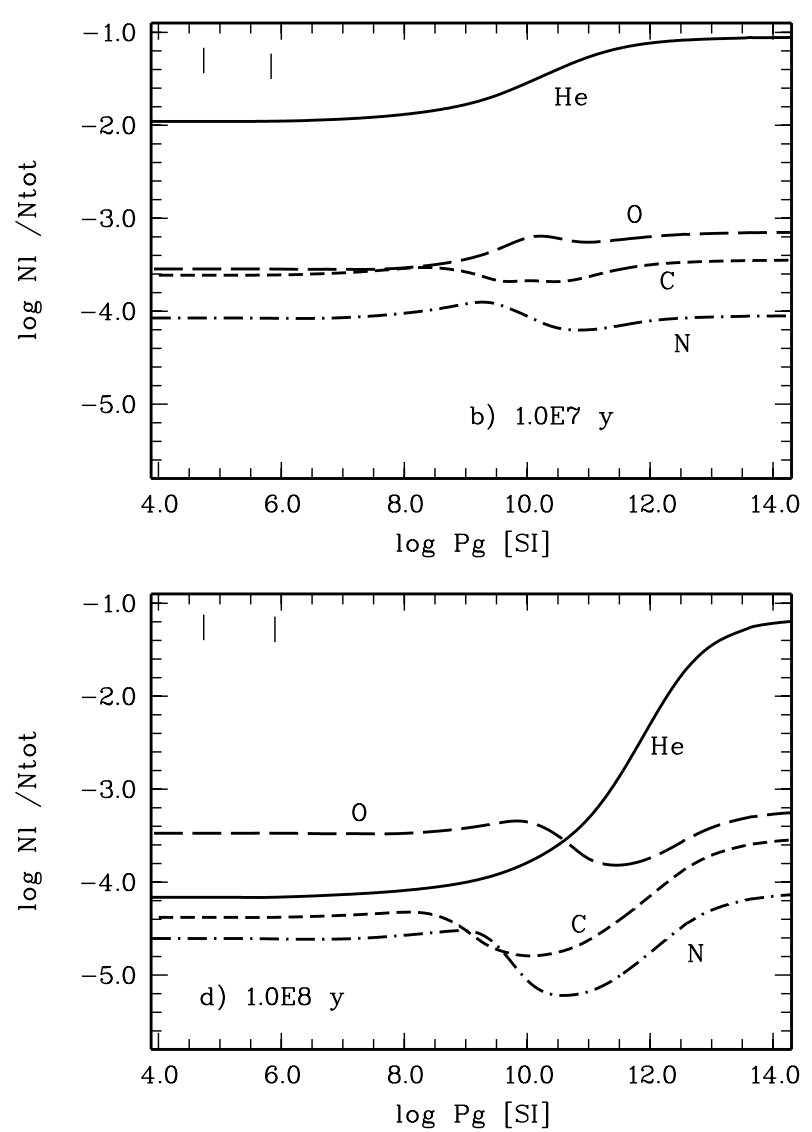

can be supplied into the regions above. If the time scale is long enough, this finally will always lead to a depletion of the element. From these results we now can explain the origin of the three stages of the time evolution of the surface abundance for $\dot{M}=2.5 \times 10^{-14} M_{\odot} /$ yr. Before an inner reservoir is present, $\mathrm{C}, \mathrm{N}$ and $\mathrm{O}$ sink. Then, when the elements are sufficiently accumulated, this trend is reversed and the surface abundance increases. Finally, when the amount of matter transported from the reservoir to the surface exceeds the supply from deeper regions, the abundance within the reservoir and as a consequence the surface abundance decreases again. As for the various elements the time scales for each of these stages differ, in course of time various abundance anomalies may occur. For helium, however, the radiative acceleration is effective in the outermost regions only, which are lost rapidly. Therefore the time dependence of the helium abundance is governed by a simple interplay between gravitational settling and mass loss. Thus helium sinks continuously, no enrichment is possible.

For comparison, in Fig. 3 the corresponding results for $\dot{M}=10^{-13} M_{\odot} /$ yr are shown. After $10^{6} \mathrm{yr}$ and $10^{7} \mathrm{yr}$ (Figs. 3a and $3 \mathrm{~b}$, respectively), within the computation domain the number fractions of $\mathrm{C}, \mathrm{N}$ and $\mathrm{O}$ do not vary by more than a factor of three. Within the whole region the abundances are almost constant. After $5 \times 10^{7} \mathrm{yr}$ and $10^{8}$ yr (Figs. 3c and 3d, respectively), near $\log P_{\mathrm{g}}=10$ and in the regions below, clear concentration gradients appear. 
However, they are much less pronounced than for the case with $\dot{M}=2.5 \times 10^{-14} M_{\odot} /$ yr. Especially no significant enrichments of $\mathrm{C}, \mathrm{N}$ and $\mathrm{O}$ are present. In no depth their abundance significantly exceeds the initial value. The reason for this is the higher mass loss rate. Instead of being accumulated, the elements are transported to the stellar surface. As a consequence, during the whole time scale of $10^{8} \mathrm{yr}$, near the stellar surface oxygen remains the most abundant one of the CNO elements, followed by carbon and nitrogen. No dramatic changes of the relative abundances of these elements occur.

From the results obtained up to now, we see that mass loss in the range $10^{-14} M_{\odot} / \mathrm{yr} \leq \dot{M} \leq 10^{-13} M_{\odot} / \mathrm{yr}$ may lead to abundance anomalies of the CNO elements and the composition changes in time scales similar to the typical ages of the sdB's near the EHB. In order to understand these results in more detail, it is instructive to compare the diffusion velocities in the various depths with the wind velocities for several mass loss rates. In Fig. 4 we have plotted the various velocities as a function of the gas pressure for solar composition. First let us consider the diffusion velocities of carbon (short dashed line), nitrogen (dashed dotted) and oxygen (long dashed). In all depths the diffusion velocities are negative (directed to the stellar interior). This means that in no depth solar abundances of the CNO elements (and helium) can be levitated by radiative forces. In deep regions $\left(\log P_{\mathrm{g}} \geq 12.0\right)$ with temperatures $T>2.5 \times 10^{6} \mathrm{~K}$ the effect of the radiative acceleration is small. Then the similar masses and charges of the various elements lead to similar diffusion velocities. The situation changes in the range $8.5<\log P_{\mathrm{g}}<12.0$. Here the diffusion velocities differ up to one order of magnitude. This is due to the influence of the radiative acceleration. It is effective because enough particles especially in the hydrogen-like ionization state are present and enough flux is carried in wavelength ranges, which can be absorbed by these ions. Finally, in an outer region characterized by $\log P_{\mathrm{g}}<8.5$ the various diffusion velocities again are of similar order of magnitude in most cases. Here the temperature is below about $4 \times 10^{5} \mathrm{~K}$ and the CNO elements have helium-like configuration or are less ionized in the outermost layers. For helium (solid line) the diffusion velocity continuously decreases towards the stellar interior. Apart from regions near the stellar atmosphere, the influence of the radiative acceleration is negligibly small for this element. Thus for helium the situation is simpler than for the heavy elements. Mass loss retards the sinking of helium, whereas no enrichment is possible, however.

The dotted lines in Fig. 4 represent the wind velocities for mass loss rates between $\dot{M}=5 \times 10^{-15} M_{\odot} /$ yr (lower line) and $\dot{M}=5 \times 10^{-13} M_{\odot} / y r$ (upper line). From a comparison of the wind velocities with the diffusion velocities some conclusion can be obtained, if the following three arguments are taken into consideration. First, in regions where the wind velocity is large in comparison to the diffusion velocities, concentration gradients tend to be leveled out. Thus these layers are in good approximation chemically homogeneous. Second, for the time dependence of

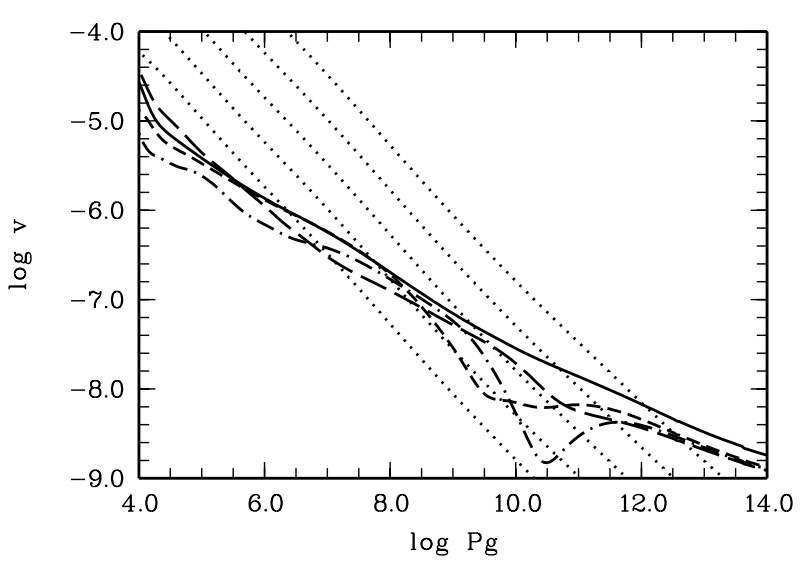

Fig. 4. For $T_{\text {eff }}=35000 \mathrm{~K}, \log g=5.5$ and solar composition the diffusion velocities (in $\mathrm{m} / \mathrm{s}$ ) of $\mathrm{He}$ (solid line), C (short dashed), $\mathrm{N}$ (dashed-dotted) and $\mathrm{O}$ (long dashed) are plotted as a function of the gas pressure (in $\mathrm{Pa}$ ). The dotted lines represent the wind velocities for various mass loss rates (from top to the bottom): $5.0 \times 10^{-13}, 1.58 \times 10^{-13}, 5 \times 10^{-14}, 1.58 \times$ $10^{-14}$ and $5 \times 10^{-15} M_{\odot} / y r$. Note that the diffusion velocities are always directed to the stellar interior, whereas the wind velocities have the opposite direction.

the surface composition, the conditions in these depths are of special importance, where the wind and diffusion velocities are of similar order of magnitude. Third, the inner regions, where the wind velocity is much smaller than the diffusion velocity, have little influence on the surface composition.

For a mass loss rate $\dot{M}=5 \times 10^{-13} M_{\odot} /$ yr the wind velocity intersects with the diffusion velocities of the $\mathrm{CNO}$ elements near $\log P_{\mathrm{g}}=12.5$ (which corresponds to a mass depth of about $10^{-4} M_{*}$ ). This is in the region where the CNO elements have similar diffusion velocities. In the overlying regions where the radiative acceleration acts, the wind velocity is much larger than the diffusion velocities. Therefore no reservoirs of the elements can be built up. Thus the surface abundances decrease continuously in time scales comparable to the diffusion time scales near the intersection point. The same would be true for larger mass loss rates, with the only difference that gravitational settling would go on even more slowly. The three intermediate dotted lines represent the wind velocities for (from top to the bottom) $1.58 \times 10^{-13}, 5 \times 10^{-14}$ and $1.58 \times 10^{-14} M_{\odot} / \mathrm{yr}$, respectively. In these cases the intersection with the diffusion velocities is in the region, where the radiative acceleration on the $\mathrm{CNO}$ elements is effective and thus the various diffusion velocities are very different. For the lower ones of these mass loss rates reservoirs can be built up similar as has been shown in Fig. 2. These metal enrichments affect the surface composition more or less and may lead to various abundance anomalies. This has the consequence that for mass loss rates in this range between about $10^{-14}$ and $10^{-13} M_{\odot} / \mathrm{yr}$ the relative abundances in the outer regions change with time. Reservoirs can build up, as has been shown in Fig. 2. For mass loss rates smaller than about $10^{-14} M_{\odot} / \mathrm{yr}$ the wind velocity 

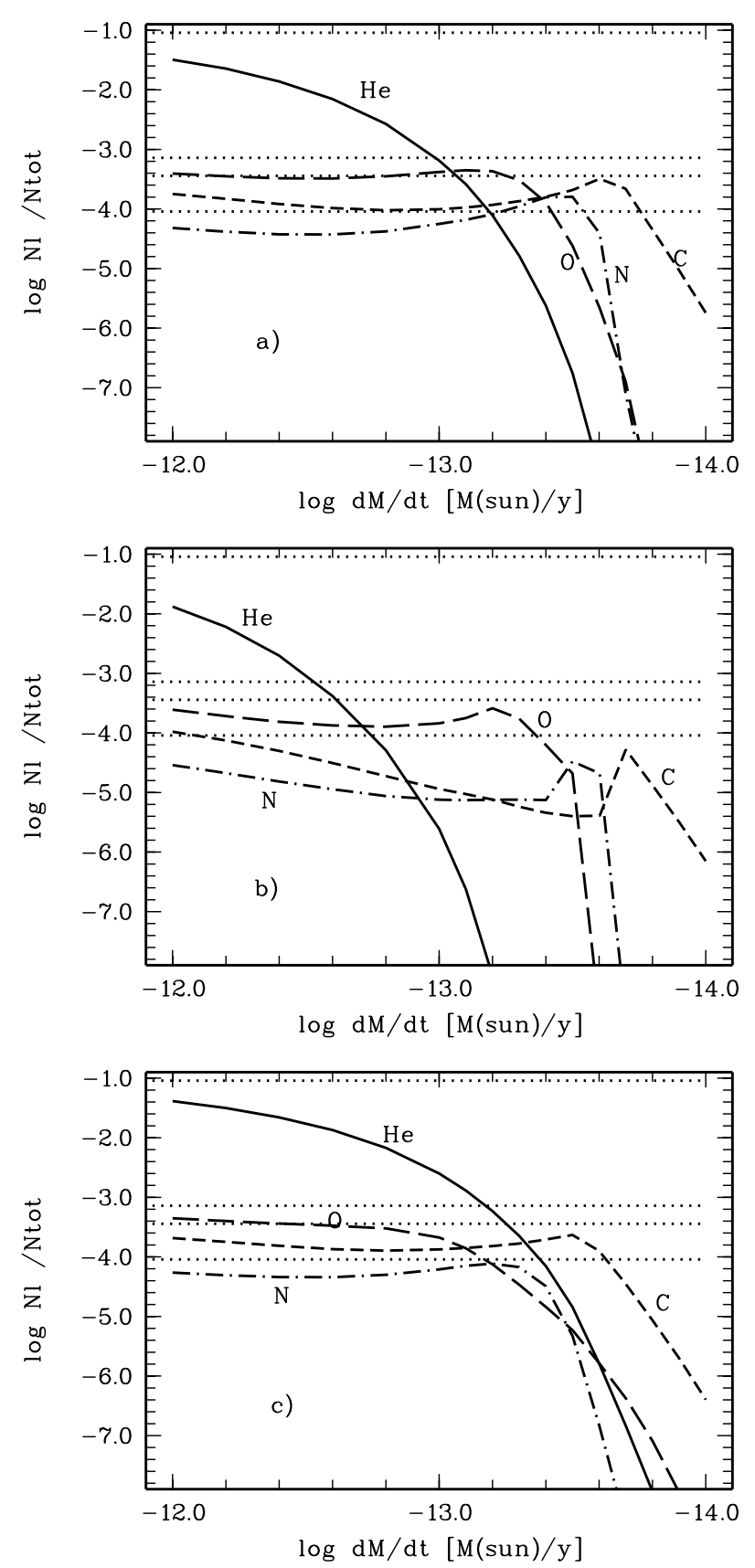

Fig. 5. The predicted surface composition after $5 \times 10^{7}$ yr as a function of the mass loss rate for a) $T_{\text {eff }}=35000 \mathrm{~K}, \log g=5.5$, b) $T_{\text {eff }}=35000 \mathrm{~K}, \log g=6.0$ and c) $T_{\text {eff }}=25000 \mathrm{~K}, \log g=$ 5.5. The dotted lines represent the solar number fractions for (from top to the bottom) He, O, C and N.

intersects with the diffusion velocity in the outer regions at $\log P_{\mathrm{g}}<8.5$. As the diffusion velocities in these depths are larger, the elements sink in time scales of a few millions of years. Large underabundances may be the consequence.

To investigate the influence of $T_{\text {eff }}$ and $\log g$ on the results, we have done several calculations in the parameter range typical for sdB's. We will not present these results in detail. Instead, in Fig. 5 the surface composition after $5 \times 10^{7} \mathrm{yr}$ is shown as a function of the mass loss rate for several cases: a) $T_{\text {eff }}=35000 \mathrm{~K}, \log g=5.5$, b) $T_{\text {eff }}=35000 \mathrm{~K}, \log g=6.0$ and c) $T_{\text {eff }}=25000 \mathrm{~K}$, $\log g=5.5$. We must have in mind that the composition changes in time, so that these are snapshots which show, however, some typical properties of the results. In all cases, for $\dot{M}=10^{-12} M_{\odot} /$ yr the abundances of the CNO elements are reduced by less than a factor of three, whereas helium is reduced by less than a factor of ten. For $\dot{M}=10^{-14} M_{\odot} /$ yr all elements are strongly depleted. The stellar atmosphere should be almost free of $\mathrm{He}, \mathrm{N}$ and O. Only some carbon may still be present with an underabundance by more than a factor of 100, however. Strong anomalies of the abundances of the CNO elements may occur for $\dot{M}<10^{-13} M_{\odot} /$ yr. Especially for the cases with $T_{\text {eff }}=35000 \mathrm{~K}$ (Figs. 5a,b) the predicted helium abundances then are below a value of $10^{-4}$, however. This is less than observed in most of the sdB's. In all cases helium should be reduced by a larger factor than the CNO elements. After $5 \times 10^{7} \mathrm{yr}$, as shown in Fig. 5 , in one case only nitrogen is the most abundant one of the CNO elements. This is for $T_{\text {eff }}=35000 \mathrm{~K}, \log g=6.0$ and $\log \dot{M} \approx-13.6$. A comparison of the results for $T_{\text {eff }}=35000 \mathrm{~K}, \log =5.5$ with those for $\log g=6.0$ shows, that for a given mass loss rate the predicted abundances of all elements are lower for the case with the higher gravity. If $T_{\text {eff }}$ is varied, whereas $\log g$ and $\dot{M}$ are fixed, no clear trends can be recognized. The maximum possible metal enrichments in the inner regions, however, which amount to about a factor of ten for $T_{\text {eff }}=35000 \mathrm{~K}, \log g=5.5$, are by about a factor of three lower in the other two cases. The results in Fig. 5 show that the predictions for a given mass loss rate in detail may be very different for the various stellar parameters. Small changes of $\dot{M}$ in some cases may lead to very different compositions. However, there is one result in common, which holds in the whole parameter range covered by the sdB's. If at all, then the typical abundances can only be explained with mass loss rates between $10^{-12}$ and $10^{-14} M_{\odot} / \mathrm{yr}$, at least if it is assumed that the star has solar composition before it evolves into an sdB. For higher mass loss rates diffusion is not effective enough to change the composition significantly, whereas for lower rates the predicted helium abundances are by far too low.

\subsection{The influence of the inner boundary condition}

A special problem are the overabundances of helium observed in a few sdB's. Within the present scenario these cannot be explained at all, if not the inner helium-rich regions have some influence on the surface composition. This could happen if helium migrates outward due to ordinary diffusion and reaches regions from which it is effectively transported to the surface by the effect of mass loss. Thus we now will investigate the influence of the lower boundary condition on the results. For $T_{\text {eff }}=35000 \mathrm{~K}, \log g=5.5$ the calculations start with the assumption of an outer hydrogen layer mass of $10^{-4} M_{*}$. According to the models of Dorman et al. (1993) the typical hydrogen layer masses in sdB's are of the order $10^{-2}$ to $10^{-3} M_{*}$. Therefore our 
choice can be considered as a lower limit, which may be realized only in objects very close to the helium main sequence or if in earlier evolutionary stages some helium-rich material would somehow be mixed into the hydrogen envelope. The calculations start with solar composition in the outer hydrogen-rich region. Below a mass depth of $10^{-4} M_{*}$ a helium- and nitrogen-rich composition is assumed with the following number ratios: $\mathrm{H} / \mathrm{He}=10^{-2}$, $\mathrm{N} / \mathrm{He}=3 \times 10^{-3}, \mathrm{C} / \mathrm{He}=\mathrm{O} / \mathrm{He}=10^{-5}$. If the star has undergone the $\mathrm{CNO}$ cycle, it is expected that the processed matter is enriched in nitrogen whereas $\mathrm{C}$ and $\mathrm{O}$ are reduced.

Now let us consider an example with a mass loss rate of $\dot{M}=5 \times 10^{-13} M_{\odot} /$ yr. With $M_{*}=0.5 M_{\odot}$ this means that within $10^{8} \mathrm{yr}$ the total mass loss is $10^{-4} M_{*}$, which is equal to the assumed hydrogen layer mass. In Fig. 6, the number fractions of the elements are plotted as a function of gas pressure for various timesteps. In Fig. 6a the situation after $10^{6} \mathrm{yr}$ is shown. It is very similar to the initial one. Near the discontinuity the effect of diffusion is small within this time scale. The same is still true after $10^{7} \mathrm{yr}$ (Fig. 6b). We recognize a beginning enrichment of $\mathrm{C}$ and $\mathrm{O}$ in the transition region between the hydrogenand helium-rich layer. This is related to the fact that in the hydrogen-rich plasma the diffusion velocities of the heavy elements are by about a factor of ten larger than in the helium-rich plasma. So on both sides of the transition region the elements sink with very different velocities. After $5 \times 10^{7}$ yr (Fig. 6c) near the stellar surface helium is still deficient and the relative abundances of the $\mathrm{CNO}$ elements are nearly unchanged. Now the tail of the $\mathrm{H} / \mathrm{He}$ transition zone has reached a depth with $\log P_{\mathrm{g}} \approx 11$, which corresponds to a mass depth of about $5 \times 10^{-6} M_{*}$. From about this depth, for the given mass loss rate helium can be effectively transported to the surface by mass loss, because here and in the regions above the wind velocity exceeds the diffusion velocity (Fig. 4). This effect stops the sinking of helium and in the following time the surface abundance gradually increases. After $10^{8}$ yr (Fig. 6d) the star has been transformed into a helium-rich subdwarf. This is an expected result, because now the total mass loss corresponds to the initially assumed hydrogen layer mass.

Figure 7 summarizes the results for this example with $\dot{M}=5 \times 10^{-13} M_{\odot} /$ yr and an initial composition discontinuity in a mass depth of $10^{-4} M_{*}$. The surface composition is plotted as a function of time. For times smaller than about $5 \times 10^{7}$ yr the abundances of helium and the CNO elements slowly decrease. Then, however, after about half of the hydrogen layer is lost, this trend reverses and the helium abundance begins to increase. After about $7 \times 10^{7} \mathrm{yr}$ the number fraction of helium exceeds the solar value until after $10^{8} \mathrm{yr}$ it is $\mathrm{H} / \mathrm{He} \approx 1$. In this special example the increase of the helium abundance is accompanied by a temporary enrichment of the heavy elements. After larger times we obtain, as expected, a surface composition similar to the one which initially has been assumed in the underlying helium-rich layer. Due to the effect of mass loss this may be kept nearly constant over large time scales. This shows that weak winds may be an effective mechanism to prevent gravitational settling and especially the floating up of hydrogen in helium-rich subdwarfs as analyzed by e.g. Lemke et al. (1997) and Bauer \& Husfeld (1995). If the mass loss rates are at least of the order $10^{-12} M_{\odot} /$ yr the effect of diffusion is small and their compositions should preferably be a consequence of their evolutionary history (see Dreizler 2001 for a review).

Similar calculations with a composition discontinuity in a mass depth of $10^{-4} M_{*}$ for lower mass loss rates, e.g. for $\dot{M}=1.0 \times 10^{-13}$ and $2.5 \times 10^{-14} M_{\odot} /$ yr, have shown that in these cases within $10^{8} \mathrm{yr}$ no effect on the surface composition can be recognized. The results are the same as shown in Figs. 1c and 1b, respectively. In addition, at least for $P_{\mathrm{g}}<10^{11} \mathrm{~Pa}$ the structure of the outer regions would be the same as shown in Figs. 3 and 2. From all these results, we expect that for mass loss rates below about $10^{-12} M_{\odot} / \mathrm{yr}$ and for the expected hydrogen layer masses of sdB's, which are usually clearly larger than $10^{-4} M_{*}$, the underlying helium-rich regions have negligible influence on the surface composition.

\subsection{Comments on the post-EHB evolution}

To investigate the possible effect of diffusion and mass loss during the post-EHB evolution, we have done some calculations during which $T_{\text {eff }}$ and $\log g$ are allowed to vary. These calculations start with models near the zero age horizontal branch with an outer hydrogen layer of $0.003 M_{\odot}$, in which initially solar composition is assumed and an underlying helium-rich region with a composition similar to the one used in Sect. 3.2. Then, with $T_{\text {eff }}$ and $\log g$ from an evolutionary track from Dorman et al. (1993) the time dependence of the composition during the EHB and post-EHB evolution has been predicted until the star has evolved into a white dwarf. Mass loss rates proportional to about the square of the luminosity have been assumed with various scaling factors. As the results may strongly depend on the mass loss rate, which is a free parameter, the conclusions are restricted to a few trends concerning the helium abundance. When during the post-EHB evolution of a helium-poor sdB the mass loss rate increases by at least a factor of 10 to 100 , the surface number fraction of helium should tendencially increase. The evolution into a helium-rich subdwarf, however, would only be possible, if mass loss removed a major part of the outer hydrogen-rich envelope. Such a scenario has been proposed by MacDonald \& Arrieta (1994) to explain a possible evolutionary link between the sdB's and helium-rich sdO's. According to our results it is not necessary, however, that all hydrogen is lost. Due to the effect of diffusion, during this process enough hydrogen should be left in the outer regions, so that after the evolution into a white dwarf hydrogen can float up again and the star evolves via the DAO stage into a DA with no detectable helium. 

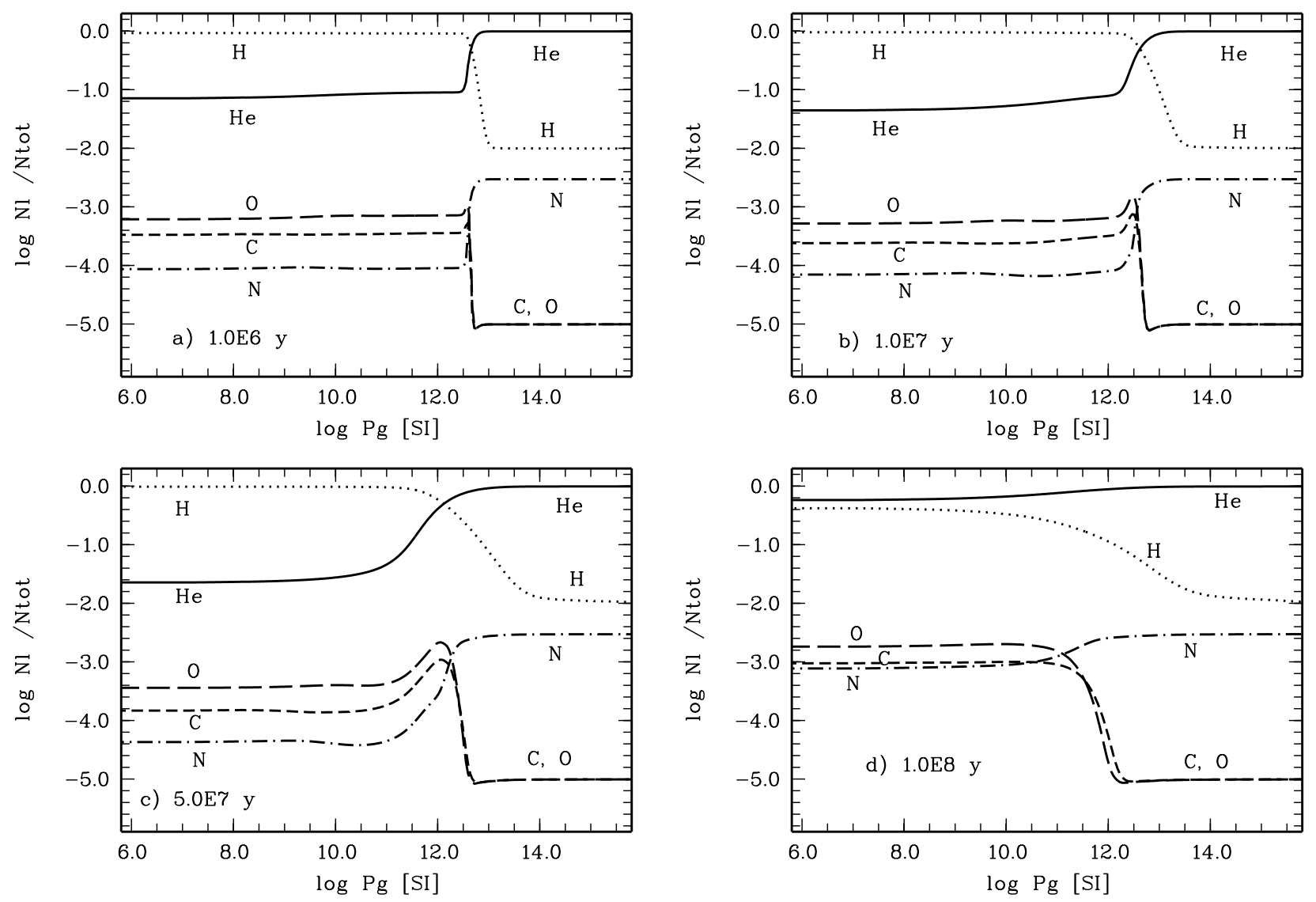

Fig. 6. Number fractions as a function of the gas pressure for the case with an initial composition discontinuity at a mass depth of $10^{-4} M_{*}$ and for $T_{\text {eff }}=35000 \mathrm{~K}, \log g=5.5, \dot{M}=5 \times 10^{-13} M_{\odot} / \mathrm{yr}$. The results after various times are plotted: a) $10^{6}$, b) $10^{7}$, c) $5 \times 10^{7}$ and d) $10^{8} \mathrm{yr}$.

\section{The existence of winds and comparison with the case of white dwarfs}

The existence of a chemically homogeneous, radiatively driven wind requires that the maximum possible radiative acceleration in the wind region is at least equal to the gravitational acceleration. For $T_{\text {eff }} \leq 50000 \mathrm{~K}$ and solar composition Abbott (1982) has calculated force multipliers, which give the radiative acceleration due to line absorption in units of the acceleration due to electron scattering. In the case of thin winds, when the lines "see" the unattenuated stellar flux, the force multiplier is of the order $10^{3}$. If this value is used for all effective temperatures, the maximum possible radiative acceleration is represented by the dashed line in the $T_{\text {eff }}-\log g$ diagram in Fig. 8. To circumvent this extrapolation of Abbott's results into regions with $T_{\text {eff }}>50000 \mathrm{~K}$, in addition we have estimated the force multipliers with the fluxes from our models (see Paper I for more details). With these values instead of a constant value of $10^{3}$, a maximum radiative acceleration corresponding to the solid line in Fig. 8 is obtained. We see that at least for $T_{\text {eff }} \leq 10^{5} \mathrm{~K}$ both predictions agree within an order of magnitude. The lines represent the wind limit. Chemically homogeneous winds may exist in the region above the wind limit only. Below the wind limit some outflow may still be present. However,

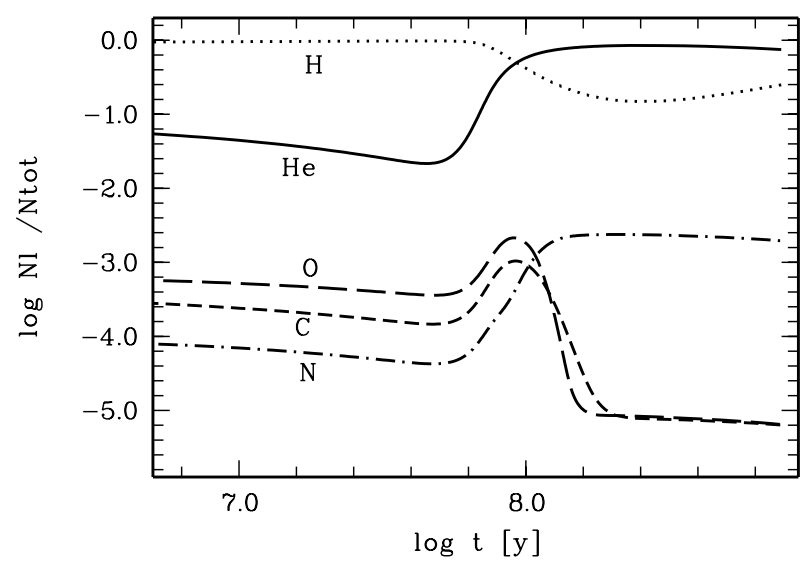

Fig. 7. For the case with an initial composition discontinuity in a mass depth of $10^{-4} M_{*}$ the surface composition is plotted as a function of time for the case with $T_{\text {eff }}=35000 \mathrm{~K}, \log g=5.5$ and $\dot{M}=5 \times 10^{-13} M_{\odot} /$ yr.

only such elements can be expelled from the photosphere for which the radiative force is sufficient, whereas others are in hydrostatic equilibrium. Such a scenario has been investigated by Babel (1995) for the case of main sequence A stars.

The filled triangles in Fig. 8 represent hydrogen-rich white dwarfs with detectable amounts of helium (DAO's) 
analyzed by Napiwotzki (1999) and Bergeron et al. (1994). We have introduced only those objects which are heliumdeficient and thus show signs of gravitational settling. The number ratios are in the range $10^{-3} \leq \mathrm{He} / \mathrm{H}<0.1$. This presence of helium cannot be explained with the assumption of an equilibrium between gravitational settling and radiative levitation. The predicted abundances are too low by at least a factor of ten, as has been shown by Vennes et al. (1988) and Unglaub \& Bues (1998). Therefore in Paper I we investigated the influence of mass loss. With $T_{\text {eff }}$ and $\log g$ from evolutionary tracks we predicted the changes of the surface abundances of helium and the CNO elements of hot white dwarfs with time. With a rough estimate of the mass loss rates, which allow for a dependence of $\dot{M}$ on the composition, at least the onset of gravitational settling has been predicted in good agreement with the observational data. From Fig. 8 it can be seen that the majority of all DAO's is above the wind limit, in the region where winds can exist. The mass loss rates must be in the range between about $10^{-11}$ and $10^{-13} M_{\odot} / \mathrm{yr}$. For higher rates no noticeable influence of gravitational settling is expected, whereas for lower rates helium would sink in time scales which are short compared to cooling ages. The existence of some DAO's below the wind limit is not in contradiction to this scenario. The radiative acceleration in the wind region and thus the location of the wind limit in the $T_{\text {eff }}-\log g$ diagram depends on the composition. This may be very different for stars which enter the white dwarf cooling sequence. Especially for the helium-, carbon- and oxygen-rich PG 1159 stars mass loss exists until later evolutionary stages as for objects with approximately "normal" composition. When they reach their wind limit, which is located at higher gravities (see Paper I), diffusion may transform them into DAO's, if sufficient hydrogen is hidden in their outer regions. Thus some of the DAO's shown in Fig. 8 below the wind limit may be in such a transition state between helium-rich white dwarfs and hydrogen-rich ones without any detectable helium (DA's). Others are special objects, which probably accrete helium from a binary companion. The open triangles in Fig. 8 represent the DA's analyzed by Schuh (2000; see also Schuh et al. 2001). The EUV fluxes of these objects in most cases can well be explained with self-consistent model atmospheres, which assume an equilibrium between gravitational settling and radiative levitation. This practically requires the absence of winds. An upper limit for the mass loss rates is $\dot{M} \approx 10^{-18} M_{\odot} / \mathrm{yr}$ (Chayer et al. 1997; Fontaine \& Chayer 1997). Thus only stars below the wind limit can be expected to be in such an equilibrium state.

The filled circles in Fig. 8 represent a sample of about 120 sdB's from the Hamburg Quasar Survey and the Palomar Green Survey analyzed by Edelmann et al. (2001). All these objects are close to the wind limit. The sdB's stay in this region for about $10^{8} \mathrm{yr}$ whereas the white dwarfs during their cooling cross the region near the wind limit within some $10^{5} \mathrm{yr}$. This is an important difference. The white dwarfs evolve from a region far above the wind

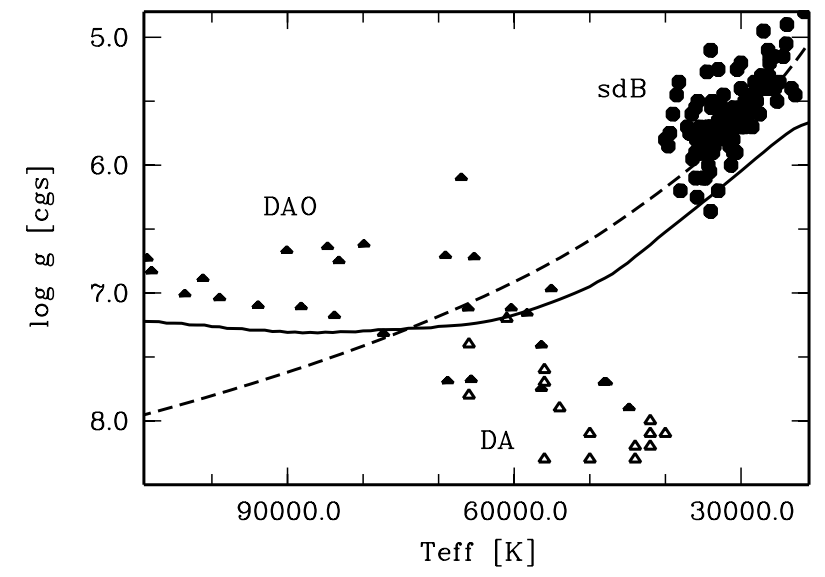

Fig. 8. The wind limit in the $T_{\text {eff }}-\log g$ diagram from our estimate (full drawn line) and for a constant force multiplier of $10^{3}$ (dashed line). In addition are shown the sdB's (filled circles) analyzed by Edelmann et al. (2001), the helium-deficient DAO's (filled triangles) analyzed by Napiwotzki (1999) and Bergeron et al. (1994) and the DA's (open circles) analyzed by Schuh (2000) with the assumption of an equilibrium between radiative levitation and gravitational settling.

limit, where strong mass loss exists and thus the effect of diffusion is negligible. Then, when they approach the wind limit, helium and the heavy elements begin to sink slowly. In this stage of evolution, the surface composition is the result of a complicated interplay between mass loss and diffusion. The depletion of elements favours the decrease of the mass loss rates. This in turn enhances the influence of gravitational settling. Then the elements sink rapidly and because of the low abundances of helium and heavy elements the mass loss rates decrease drastically. At this stage of evolution DAO's transform into DA's. For the majority of the DAO's our predictions in Paper I can reproduce the relation between the luminosities and the helium abundances found by Napiwotzki (1999). Thus diffusion and mass loss appear to be a very likely explanation of the chemical evolution of hot white dwarfs on the upper cooling sequence. In the case of sdB's, the situation is more complicated insofar, as they are exposed to the interplay of diffusion and mass loss over much larger times. This leads to interesting effects like metal enrichments. The abundances depend on the time the star has spent on the EHB. Thus objects with similar stellar parameters may have different compositions. If the abundances are plotted as a function of the luminosity, $T_{\text {eff }}$ or $\log g$, a wide spread must be expected. This may be the reason why no clear dependence of the helium abundance on $T_{\text {eff }}$ and $\log g$ can be recognized (Schulz et al. 1991; Fontaine \& Chayer 1997; Edelmann et al. 2001). If the mass loss rates preferably depends on the luminosity, more luminous objects should tendencially have higher helium abundances. However, in contrast to the white dwarfs on the upper cooling sequence, for which the luminosities vary by several orders of magnitude, the luminosities of most sdB's are between 10 and $100 L_{\odot}$. Therefore and because of the expected large scatter a possible relation between the 
luminosity and the composition can hardly be detected. Thus in the case of sdB's a comparison of the predictions with observational results is more involved as for white dwarfs.

Winds which are weak enough to allow for an interplay between mass loss and diffusion can hardly be detected and theoretical predictions of the mass loss rates do not exist. Thus we must rely on rough estimates or extrapolate the results obtained for dense winds. From Abbott's (1982) formula, we obtain mass loss rates of the order $10^{-13} M_{\odot} / \mathrm{yr}$ for $T_{\text {eff }}=30000 \mathrm{~K}, \log g=6.0$ and $10^{-11} M_{\odot} / \mathrm{yr}$ for $T_{\text {eff }}=35000 \mathrm{~K}, \log g=5.5$. In Paper I we roughly estimated $\dot{M}$ from the conditions at the sonic point, where the wind speed is equal to the isothermal sound velocity. With this method we obtain $\dot{M}=7 \times 10^{-14} M_{\odot} / \mathrm{yr}$ for $T_{\text {eff }}=30000 \mathrm{~K}, \log g=6.0$ and $\dot{M}=10^{-12} M_{\odot} /$ yr for $T_{\text {eff }}=35000 \mathrm{~K}, \log g=5.5$. However, all these methods or extrapolations are problematical for at least two reasons. First, in thin winds the radiative acceleration may strongly depend on the velocity (Babel 1996). Thus methods similar to the one of Castor et al. (1975), in which only the dependence on the velocity gradient is taken into account, may considerably overestimate the mass loss rate. Second, Springmann \& Pauldrach (1992) suggest that for thin winds a multifluid description is required. As the Coulomb coupling between radiatively accelerated ions and the field particles (e.g. protons) is not sufficiently efficient, a decoupling of the various species of particles may occur. How this may affect the mass loss rate and the chemical composition of these stars, has been investigated only by Babel (1995) for main sequence A stars. It appeared that the decoupling may under certain circumstances lead to enrichments or deficiencies of elements in the photosphere. Possibly such a scenario is an important alternative explanation of the anomalies observed in sdB's.

\section{Summary and conclusions}

In the present paper it has been shown what the combined effects of mass loss and diffusion can do. If at all the observed abundances can be explained in this way, this is only possible with mass loss rates in the range $10^{-14} M_{\odot} / \mathrm{yr} \leq \dot{M} \leq 10^{-12} M_{\odot} /$ yr. Higher rates would effectively prevent the effect of diffusion, whereas for lower rates helium would sink in time scales less than $10^{7} \mathrm{yr}$, which is much less than the typical lifetimes of the sdB's near the EHB. The helium abundances could be explained with weak winds of the order $\dot{M} \approx 10^{-13} M_{\odot} / \mathrm{yr}$. Then within a typical lifetime of $10^{8}$ yr the number fraction decreases from the solar value to about $10^{-4}$. This covers the typical range of the observed values. Whereas the relative abundances of the CNO elements remain almost unchanged for cases with $\dot{M}>10^{-13} M_{\odot} /$ yr, strong anomalies may occur for lower mass loss rates. Diffusion and mass loss in the course of time may lead to enrichments of these elements as well as to strong deficiencies by more than a factor of 100. In regions with mass depths of the order
$10^{-6}$ to $10^{-8} M_{*}$, within time scales of $10^{7}$ to $10^{8} \mathrm{yr}$ reservoirs of the CNO elements may build up, within which the abundances are up to a factor of ten above the solar value. This is possible although such abundances cannot be levitated by the radiative acceleration. Due to the coupling between internal and surface composition in the presence of mass loss, these reservoirs lead to varying surface compositions in similar time scales. Because of this time dependence, sdB's with similar stellar parameters may have very different compositions. These results support the suggestion of Charpinet et al. (1999) that the presence of weak stellar winds may be responsible for varying metal abundances near the potential driving region of pulsations. Such variations would be an obvious explanation for the coexistence of pulsating and non-pulsating sdB stars in the same area of the $\log g-T_{\text {eff }}$ diagram.

The results show that diffusion and mass loss possibly can explain the helium abundances and may lead to strong anomalies of heavy elements as well as to metal enrichments in the outer envelope, which is of importance for an explanation of the pulsation mechanism. However, there are some observational hints that the real situation is more complicated. According to the predictions, in each case helium should be reduced by a larger factor than the CNO elements. This is plausible because of the influence of the radiative acceleration on the heavy elements, whereas in the case of helium their effect is negligible. Moreover, even such elements for which the radiative acceleration is less effective probably should sink more slowly than helium. This is because the resistance coefficients are proportional to the square of the mean charge of an element. Thus, although metals are heavier than helium, which favours gravitational settling, because of their higher charges their diffusion velocities should be tendencially lower. The model atmosphere analysis of sdB's carried out up to now show, however, that in some cases various heavy elements (e.g. $\mathrm{C}$ and $\mathrm{O}$ ) are clearly more reduced (in comparison to the solar value) than helium (e.g. Heber et al. 2000). According to our predictions this should not be possible. A second problem is that in most sdB's nitrogen is the most abundant one of the CNO elements. Within a small range of mass loss rates this may indeed be possible in some cases. However, the predictions cannot explain why there should be a preference for nitrogen. Instead we expect a variety of several anomalies.

In spite of these discrepancies we suggest that the abundance patterns of sdB's are related to the effects of diffusion and mass loss. This is because of the vicinity of the sdB's to the wind limit. As discussed in Sect. 4, in stars near the wind limit the combined effects of mass loss and diffusion are likely to be effective. On the one hand, the mass loss rates are likely to be low enough so that mass loss cannot entirely prevent the effect of diffusion. On the other hand, the weak outflow which still exists would destroy the abundance profile predicted by equilibrium diffusion calculations. In such cases we are confronted with a complicated situation. In the following we discuss 
some possible reasons for the discrepancies between our predictions and observed abundances in sdB's:

1. The Coulomb coupling between the various species of particles in the wind region is possibly not sufficent to redistribute the momentum absorbed from photons efficiently over all species. Thus the separation of elements may occur either in regions deep below the stellar atmosphere as discussed in the present paper or in the wind region. The latter possibility should be investigated in the future.

2. Even if the winds are chemically homogeneous as assumed in the present investigation, the varying composition probably will lead to a varying mass loss rate. In addition, during the evolution of the sdB's near the EHB $T_{\text {eff }}$ and $\log g$ change with time.

3. In view of the results of Charpinet et al. (1997) iron should contribute significantly to the opacity in the outer envelope of sdB's. A steeper temperature gradient would probably change the location of the metal enriched reservoirs and thus the predicted surface composition.

4. If, for example due to the possible metal enrichments, certain regions in the outer envelope are convectively unstable this could change the surface composition even if the convection zone does not reach up to the surface. Mixing processes tendencially should slow down the sinking of helium and lead to a more smooth concentration gradient between the inner and outer regions. For the heavy elements, however, the consequences are less clear as mixing may destroy the metal enriched reservoirs.

5. The results depend on the initial composition assumed in the outer envelope.

Especially the latter point deserves attention. In the presence of mass loss the surface composition more or less will exhibit signatures of the abundance distribution in the outer envelope at the beginning of the EHB evolution. For high mass loss rates the initial composition remains nearly unchanged, whereas for decreasing rates the abundances are more and more modified by diffusion. Only in the case of negligible mass loss the abundances of trace elements can be predicted in good approximation as a function of $T_{\text {eff }}$ and $\log g$, independently of the history of the star. This is done in equilibrium diffusion calculations. For the mass loss rates considered in the present paper, the initial composition in the outer regions with mass depths smaller than about $10^{-4} M_{*}$ may affect the results. Thus dredge up of processed material in previous stages of evolution as well as differences in the metallicity of the matter out of which the sdB's have been formed may be of significance. The origin of the sdB's is still under discussion. From the several binary and single star scenarios especially the "helium mixing scenario" proposed by Sweigart (1997) is of interest, according to which mixing processes in the red giant phase of evolution not only changes the relative abundances of various heavy elements in the envelope, but in addition lead to an enrichment of helium. This could be a possible explanation of the overabundances of helium observed in a few sdB's, which in no case can be explained by our calculations, if initially a thick hydrogen layer with a solar $\mathrm{He} / \mathrm{H}$ ratio is assumed. In addition, the mixing of matter processed by the CNO cycle would lead to increased $\mathrm{N} / \mathrm{C}$ and $\mathrm{N} / \mathrm{O}$ ratios. Thus the abundances of these elements observed in sdB's may be partially due to nucleosynthesis. This could explain why nitrogen is in most cases more abundant than carbon and oxygen. During the EHB evolution the various abundances will be modified by diffusion, dependent on how large the mass loss rate is.

Acknowledgements. We thank R. Napiwotzki and the referee for carefully reading the manuscript and useful comments which helped to improve the paper. U. Heber and H. Edelmann are thanked for making unpublished results of model atmosphere analysis available and for answering many questions.

\section{References}

Abbott, D. C. 1982, ApJ, 259, 282

Babel, J. 1995, A\&A, 301, 823

Babel, J. 1996, A\&A, 309, 867

Baschek, B., Höflich, P., \& Scholz, M. 1982a, A\&A, 112, 76

Baschek, B., Kudritzki, R. P., Scholz, M., \& Simon, K. P. 1982b, A\&A, 108, 387

Bauer, F., \& Husfeld, D. 1995, A\&A, 300, 481

Bergeron, P., Wesemael, F., Beauchamp, A., et al. 1994, ApJ, 432, 305

Bergeron, P., Wesemael, F., Michaud, G., \& Fontaine, G. 1988, ApJ, 332, 964

Burgers, J. M. 1969, Flow Equations for Composite Gases (Academic Press, New York)

Castor, J. I., Abbott, D. C., \& Klein, R. I. 1975, ApJ, 195, 157

Charpinet, S., Fontaine, G., Brassard, P., \& Dorman, B. 1996 ApJ, 471, L103

Charpinet, S., Fontaine, G., Brassard, P., et al. 1997, ApJ, 483, L123

Charpinet, S., Fontaine, G., Brassard, P., et al. 1999, ASP Conf. Ser., 169, 166

Chayer, P., Fontaine, G., \& Pelletier, C. 1997, in White Dwarfs, ed. J. Isern, M. Hernanz, \& E. Garcia-Berro (Kluwer Academic Publishers), 253

Clark, R., Cowan, R., \& Bobrowicz, F. 1986, Atom. Data Nuclear Tables, 34, 415

Dorman, B., Rood, R. T., \& O'Connell, R. W. 1993, ApJ, 419, 596

Dreizler, S. 2001, Encyclopedia of Astronomy and Astrophysics, submitted

Edelmann, H., Heber, U., Napiwotzki, R., et al. 2001, A\&A, in preparation

Fontaine, G., \& Chayer, P. 1997, The Third Conference on Faint Blue Stars, ed. A. G. D. Philip, J. Liebert, \& R. A. Saffer (Davis Press), 169

Heber, U. 1986, A\&A, 155, 33

Heber, U. 1998, in Ultraviolet Astrophysics - Beyond the IUE Final Archive, ESA SP-413, 195

Heber, U., Hamann, W. R., Hunger, K., et al. 1984, A\&A, 136, 331

Heber, U., Reid, I. N., \& Werner, K. 1999, A\&A, 348, L25

Heber, U., Reid, I. N., \& Werner, K. 2000, A\&A, 363, 198 
Lamontagne, R., Wesemael, F., Fontaine, G., \& Sion, E. M. 1985, ApJ, 299, 496

Karzas, W. J., \& Latter, R. 1961, ApJS, 6, 55

Lamontagne, R., Wesemael, F., \& Fontaine, G. 1987, ApJ, 318, 844

Lemke, M., Heber, U., Napiwotzki, R., Dreizler, S., \& Engels, D. 1997, The Third Conference on Faint Blue Stars, ed. A. G. D. Philip, J. Liebert, \& R. A. Saffer (Davis press), 375

MacDonald, J., \& Arrieta, S. S. 1994, in Proc. Hot Stars in the Halo, ed. S. J. Adelman, A. R. Upgren, \& C. J. Adelman (Cambridge University Press), 238

Massacrier, G. 1996, A\&A, 309, 979

Maxted, P. F. L., Heber, U., Marsh, T. R., \& North, R. C. 2001, MNRAS, submitted

Michaud, G., Bergeron, P., Heber, U., \& Wesemael, F. 1989, ApJ, 338, 417

Moehler, S., Heber, U., \& de Boer, K. S. 1990, A\&A, 239, 265

Napiwotzki, R. 1999, A\&A, 305, 101

O'Donoghue, D., Koen, C., Kilkenny, D., Stobie, R. S., \& Lynas-Gray, A. E. 1999, ASP Conf. Ser., 169, 149

Ohl, R. G., Chayer, P., \& Moos, H. W. 2000, ApJ, 538, L95

Paquette, C., Pelletier, C., Fontaine, G., \& Michaud, G. 1986a, ApJS, 61, 177
Paquette, C., Pelletier, C., Fontaine, G., \& Michaud, G. 1986b, ApJS, 61, 197

Rauch, T. 1993, A\&A, 276, 171

Saffer, R. A. Bergeron, P., Koester, D., \& Liebert, J. 1994, ApJ, 432, 351

Schuh, S. 2000, Diploma Thesis, University of Tübingen

Schuh, S., Dreizler, S., \& Wolff, B. 2001, Proc. of the 12th European Workshop on White Dwarfs, ed. H. L. Shipman, \& J. L. Provencal, ASP Conf. Ser., in press

Schulz, H., Heber, U., \& Wegner, G. 1991, PASP, 103, 435

Springmann, U. W. E., \& Pauldrach, A. W. A. 1992, A\&A, 262,515

Sweigart, A. 1997, The Third Conference on Faint Blue Stars, ed. A. G. D. Philip, J. Liebert, \& R. A. Saffer (Davis press), 3

Unglaub, K., \& Bues, I. 1996, A\&A, 306, 843

Unglaub, K., \& Bues, I. 1997, A\&A, 321, 485

Unglaub, K., \& Bues, I. 1998, A\&A, 338, 75

Unglaub, K., \& Bues, I. 2000, A\&A, 359, 1042 (Paper I)

Vauclair, G., Vauclair, S., \& Greenstein, J. L. 1979, A\&A, 80, 79

Vennes, S., Pelletier, C., Fontaine, G., \& Wesemael, F. 1988, ApJ, 331, 876 